\title{
Probe-Based Confocal Laser Endomicroscopy of the Lungs
}

\author{
Meng Peng ${ }^{1}$, Tan Gan Liang ${ }^{2}$ and Devanand Anantham ${ }^{1,2^{\star}}$ \\ ${ }^{1}$ Duke-NUS Graduate Medical School, Singapore \\ ${ }^{2}$ Department of Respiratory and Critical Care Medicine, Singapore General Hospital, Singapore
}

\begin{abstract}
Probe-based confocal laser endomicroscopy (pCLE) is a relatively new technology in pulmonary medicine. Real time, in vivo visualization of elastin microstructure in the airway wall as well as alveoli is possible via flexible bronchoscopy under moderate sedation. This technique delivers high quality images without significant morbidity, and potentially enables non-invasive diagnosis. The images acquired have low intraobserver and intrapatient variability. The structure or normal airways and alveoli have already been elucidated. PCLE has been used in pilot studies for the diagnosis of various malignant lesions, airway diseases and diffuse parenchymal lung diseases. There may also be a role in monitoring of treatment response and disease progression. However, development is still needed both in the technology and standardization of endoscopic procedure to realize the full potential of pCLE.
\end{abstract}

Keywords: Optical biopsy; Probe-based confocal laser endomicroscopy (pCLE); Flexible bronchoscopy; Malignant lesions; Airway diseases; Diffuse parenchymal lung diseases

\section{Introduction}

Optical biopsies using in vivo imaging at the microscopic level have opened up the possibility of non-invasive tissue sampling. Probebased confocal laser endomicroscopy (pCLE) via flexible bronchoscopy is an emerging technology that may make this a reality. The confocal microscope was first patented by Minsky in 1957 [1]. It utilizes point illumination and a pinhole apparatus to block out-of-focus rays of light, enabling the generation of high-resolution images with a lateral resolution of $5 \mu \mathrm{m}$. Started from 1980's, it has been used in vivo to image organs such as eye, skin and oral cavity. With the emerging technique of fiber based confocal microscopy, it has become small enough to enter body cavities such as the gastrointestinal tract, bladder, cervix and now the lungs [2]. In gastrointestinal endoscopy, pCLE is considered non-inferior to standard biopsy for Barrett's esophagus and there are consensus recommendations for its use in the detection of gastrointestinal malignancies, as well as inflammatory bowel diseases [3]. This review will describe how pCLE is performed as part of bronchoscopic evaluation, explain image interpretation and provide an update on emerging clinical indications in pulmonary diseases.

\section{How to Perform pCLE}

There are currently two commercially available confocal endomicroscope systems for thoracic imaging [4]. Optiscan Five $1^{\circ}$ (Optiscan PTY Ltd, Victoria, Australia) utilizes a $5 \mathrm{~mm}$ to $6.3 \mathrm{~mm}$ rigid probe that can be used during thoracoscopy and rigid bronchoscopy. However, it cannot be passed through the working channel of a standard flexible bronchoscope [5]. Cellvizio (Mauna Kea Technologies, Paris, France) utilizes a $1.4 \mathrm{~mm}$ semi-flexible bronchoscopic miniprobe (Figure 1) that can be used in flexible bronchoscopy under moderate sedation. This miniprobe generates a $600 \mu \mathrm{m} \times 600 \mu \mathrm{m}$ field of view under the illumination of a laser source with an excitation wavelength of $488 \mathrm{~nm}$. Images are acquired at 12 frames per second and the $\mathrm{z}$-depth is fixed at $50 \mu \mathrm{m}$. The lateral resolution is $3.5 \mu \mathrm{m}$. This review will focus on the data available on the bronchoscopic miniprobe.

Prior to bronchoscopy the pCLE processor requires an initialization process that takes 2 minutes. The small field of view makes systematic examination of the entire airways impractical. Therefore, white light bronchoscopy is performed first before a target area is scanned with pCLE. Images are obtained by gentle perpendicular application of the miniprobe directly against the mucosa. For optimal imaging, secretions must first be suctioned out and the airways adequately anaesthetized with topical lidocaine. Further processing of video sequences is possible with an algorithm that uses data fitting to 'stitch' together a mosaic. This algorithm combines successive images, as well as cancels movementinduced artifacts to reconstitute a widened field of view. A mosaic is created by gently drawing the probe across the target and the image area can be increased 2 -fold to 4 -fold.

Examination of distal airspaces should be attempted after detailed review of thoracic computed tomography imaging and performed with the smallest available bronchoscope (outer diameter $4.9 \mathrm{~mm}$ in the authors' institution) to facilitate access to a targeted bronchopulmonary segment. Usually up to eight segments are scanned and only unilateral examination is performed because of the potential for pneumothorax. For correlation with radiology, it is necessary to rigorously document the segment that is entered. Scanning of the apical and posterior segment of the upper lobes is not always possible because of the stiffness of the pCLE miniprobe. Once the bronchoscope is placed in the smallest accessible bronchus, the miniprobe is gently advanced until it passes through bronchiolar walls before reaching the alveolar acini. Typically en-face images are obtained but images at different angles provide various perspectives. Once the alveoli are reached, the miniprobe pressing on more distal structures causes a compression effect. This visualizes background planes beyond the $50 \mu \mathrm{m}$ focus creating a 3-dimensional effect. Best results are obtained by withdrawing the probe once acini are seen and analyzing the last image before contact is lost [6] (Figure 2). At this point alveolar deformation is minimized and reproducible measurements are possible. When clear images are seen, videos of up to 30 seconds are recorded in each targeted area.

Post bronchoscopy, videos are reviewed and representative still pictures are obtained for analysis. The viewer software facilitates

${ }^{*}$ Corresponding author: Devanand Anantham, Department of Respiratory and Critical Care Medicine, Academia Building Level 3, Singapore General Hospital, Outram Road-169608, Singapore, Tel: 65-63214700; Fax: 65-62271736; E-mail: anantham.devanand@singhealth.com.sg

Received July 23, 2015; Accepted October 19, 2016; Published October 22 2016

Citation: Peng M, Liang TG, Anantham D (2016) Probe-Based Confocal Laser Endomicroscopy of the Lungs. J Pulm Respir Med 6: 373. doi: 10.4172/2161105X.1000373

Copyright: ( $) 2016$ Peng M, et al. This is an open-access article distributed under the terms of the Creative Commons Attribution License, which permits unrestricted use, distribution, and reproduction in any medium, provided the original author and source are credited. 
Citation: Peng M, Liang TG, Anantham D (2016) Probe-Based Confocal Laser Endomicroscopy of the Lungs. J Pulm Respir Med 6: 373. doi: $10.4172 / 2161-105 X .1000373$

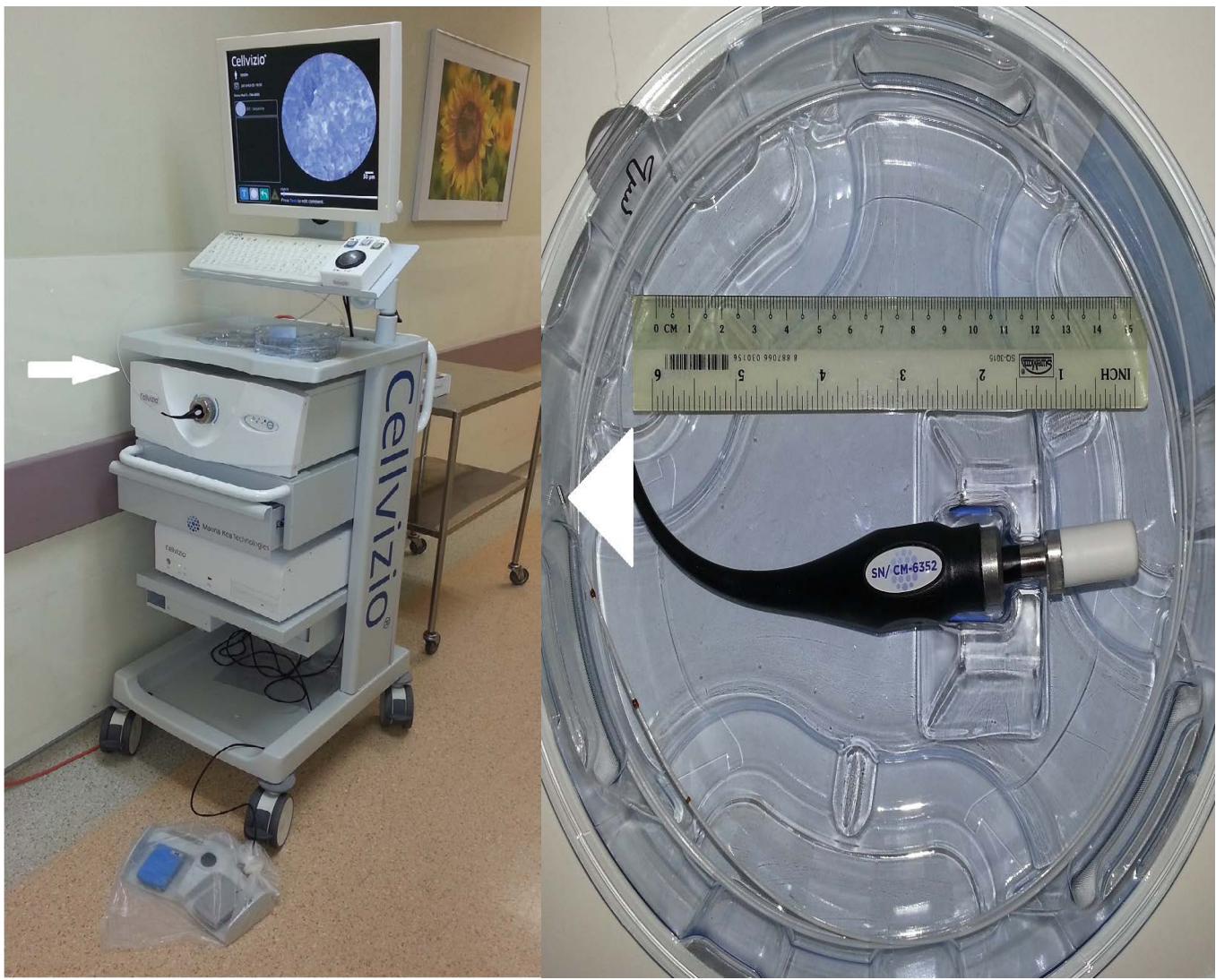

Figure 1: Probe-based confocal laser endomicroscope system utilizing a bronchoscopic miniprobe. (A: White arrow indicates the probe; B: White arrow head indicates the tip of the probe).

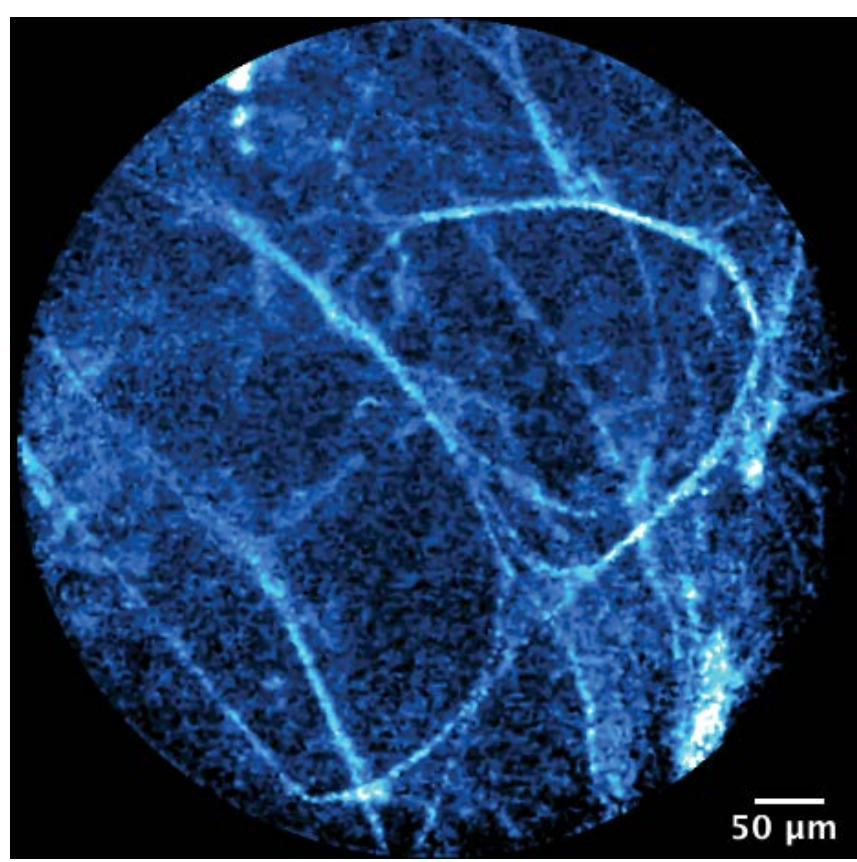

Figure 2: Normal alveolar acini captured just prior to loss of contact with the miniprobe.

measurement of alveolar structure dimensions and fluorescence intensity. Figure 3 shows how images and video clips are systematically evaluated at the authors' institution. Intra-observer (intraclass correlation coefficient i.e. ICC ranging from 0.69 to $0.91 ; \mathrm{p}<0.001)$ and intra-patient (ICC ranging from 0.65 to $0.84 ; \mathrm{p}<0.001$ ) reliability have been proven to be excellent [7]. Inter-observer agreement between blinded reviewers on the brightness of images has been shown to be high with an ICC ranging from 0.53 to $0.99(\mathrm{p}<0.001)$. However, the agreement on fiber thickness in the respiratory bronchioles is poor with an ICC of $0.12(\mathrm{p}<0.05)$ and in the alveoli is fair with an ICC of 0.37 to $0.42(\mathrm{p}<0.001)$ [7].

The reported complication rate of pCLE is low. One reported case of pneumothorax recovered spontaneously without chest tube insertion or hospitalization [8]. In addition, the visceral pleural surface has characteristic pCLE imaging of cross hatched fluorescent linear structures that can be used to identify the limit of advancing the miniprobe [9]. There is no current recommendation for the use of fluoroscopy to prevent pneumothorax. Although most studies have performed pCLE on only one side, preliminary data suggest that it can even be performed safely bilaterally [10]. Other complications of minor bleeding and transient pain have also been reported [6,11]. Occasional desaturations have occurred in authors' experience. Even patients with emphysema, pulmonary fibrosis and lung transplantation can tolerate the procedure safely with minimal discomfort and no additional sedation [12,13]. It is estimated that pCLE adds approximately 10 minutes to flexible bronchoscopy duration $[6,11]$.

\section{Image Interpretation}

After excitation with light at $488 \mathrm{~nm}$, the predominant fluorescence emission in the lungs originates from elastin as demonstrated by in vivo spectral analysis [14]. Collagen and intracellular flavins emit an 


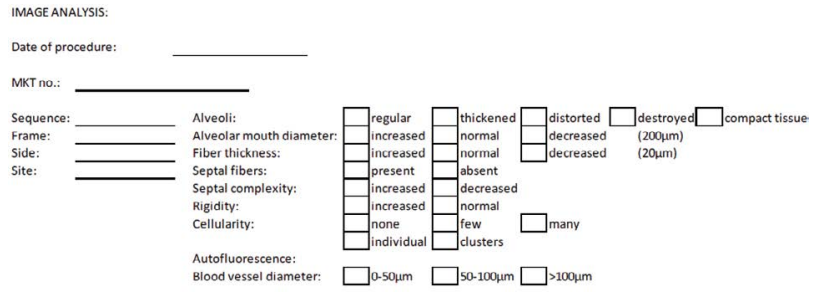

Figure 3: Checklist for image interpretation.

extremely weak signal and cannot be seen. Elastin is a component of the subepithelial layer of the airways (Figure 4) and the scaffolding of pulmonary acini (Figure 2) where it is concentrated at the alveolar duct rims, as well as the alveolar entrances $[14,15]$. Elastin is also a component of the external sheath of small blood vessels [14]. Intensity of fluorescence from elastin increases with age, presumably related to cross-linking that occurs with time [16]. In addition, fluorescence is seen in the alveolar macrophages of smokers (Figure 5) as confirmed by bronchoalveolar lavage [17]. The number, size and mobility of these fluorescent macrophages are directly correlated to the quantity smoked per day and in vitro evidence shows that the fluorescence is attributed to the particulate fraction of cigarette smoke especially tobacco tar [6].

Various images can be seen depending on the location within the bronchial tree [14]. At the anterior wall of the trachea and main stem bronchi, a dense homogenous pattern without crossing fibers is seen in Figure 6. At the orifices of the lobar bronchi, a network of tightly compacted, crossing fibers is found. This pattern is also seen around bronchial gland openings, which are $100 \mu \mathrm{m}$ to $200 \mu \mathrm{m}$ in diameter (Figure 7). The rest of the bronchi have the classical mat pattern of longitudinal $10 \mu \mathrm{m}$ fibers that are crossed perpendicularly by thin fibers. At the bronchiolar level, an interlacing lattice of $2 \mu \mathrm{m}$ to $5 \mu \mathrm{m}$ fibers is seen in Figure 8. Terminal bronchioles are identified by a helicoidal pattern, which can be transiently seen as the endoscopist penetrates into the alveolar acini. In carcinoma-in-situ and severe airway dysplasia, the regular fibers appear disorganized or may not even be seen [14]

In the distal airspaces, pCLE visualizes the axial framework of the alveolar ducts. Alveolar entrance diameters are normally distributed with a mean size of $278 \mu \mathrm{m} \pm 53 \mu \mathrm{m}$, but appear smaller in the upper lobes and right medial basal segment (paracardiac region) [6]. This may be explained by reduced ventilation to these segments in the supine position. Healthy individuals have a mean axial fiber thickness of 10 $\mu \mathrm{m} \pm 2.7 \mu \mathrm{m}$ with no measured differences between smokers and nonsmokers [6]. Small vessels are distributed throughout the lung with a mean diameter of $90 \mu \mathrm{m} \pm 50 \mu \mathrm{m}$ (Figure 9) [6]. Minor trauma can be expected given the relative size of the miniprobe compared to terminal bronchioles. Therefore, free swaying septal walls and severing of microvessels can be seen even in normal lungs.

\section{Emerging Clinical Applications}

\section{Malignant lesions}

The pCLE can be used to screen central airways for cancer. Without the administration of contrast agents, airway epithelial cells cannot be seen. Disappointingly, intravenous fluorescein sodium has not been able to demonstrate cellular structures in central airways [18]. However, using the gastrointestinal probe, which has a lateral resolution of $1.0 \mu \mathrm{m}$ and $\mathrm{z}$-depth of $65 \mu \mathrm{m}$, administration of intravenous $10 \%$ fluorescein was able to demonstrate that squamous cell carcinoma had increased cellular densities, irregular stratified patterns and capillary neovascularization [19]. Adverse effects have been primarily related to the mild allergic properties of fluorescein [4].

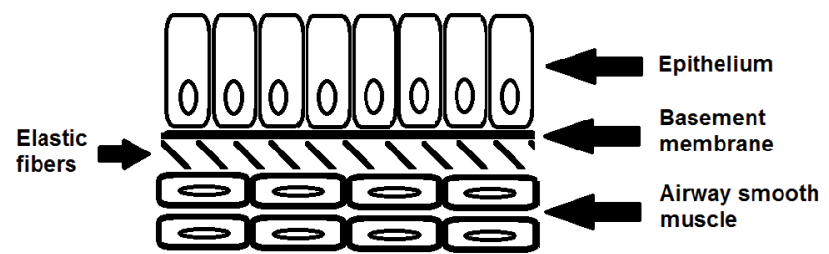

Figure 4: Schematic figure showing microscopic cross section of the airway wall (not drawn to scale).

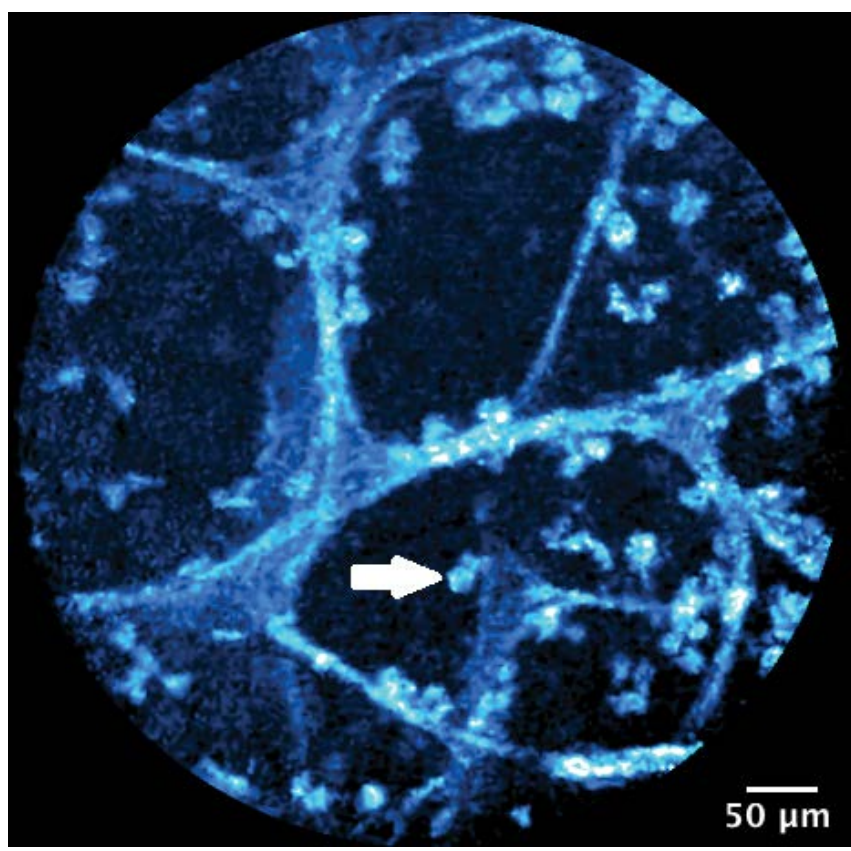

Figure 5: Alveolar macrophages (white arrow) in a smoker's lung.

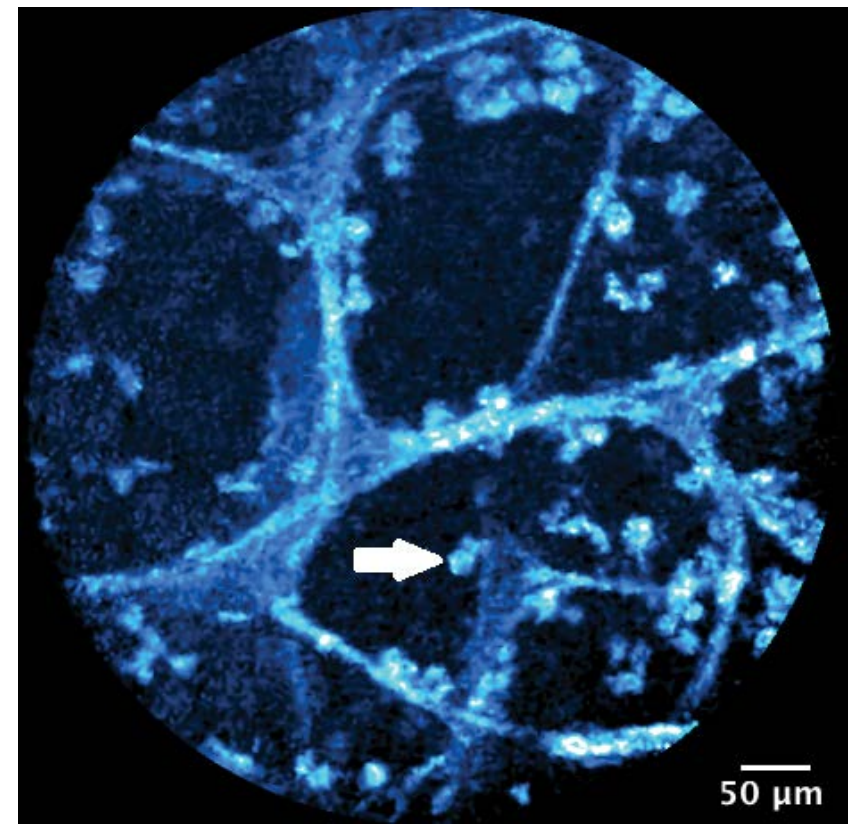

Figure 6: Anterior wall of the trachea and the main stem bronchi with a dense homogenous pattern and of fibers. No crossing fibers are seen. 
Citation: Peng M, Liang TG, Anantham D (2016) Probe-Based Confocal Laser Endomicroscopy of the Lungs. J Pulm Respir Med 6: 373. doi: 10.4172/2161-105X.1000373

Page 4 of 7

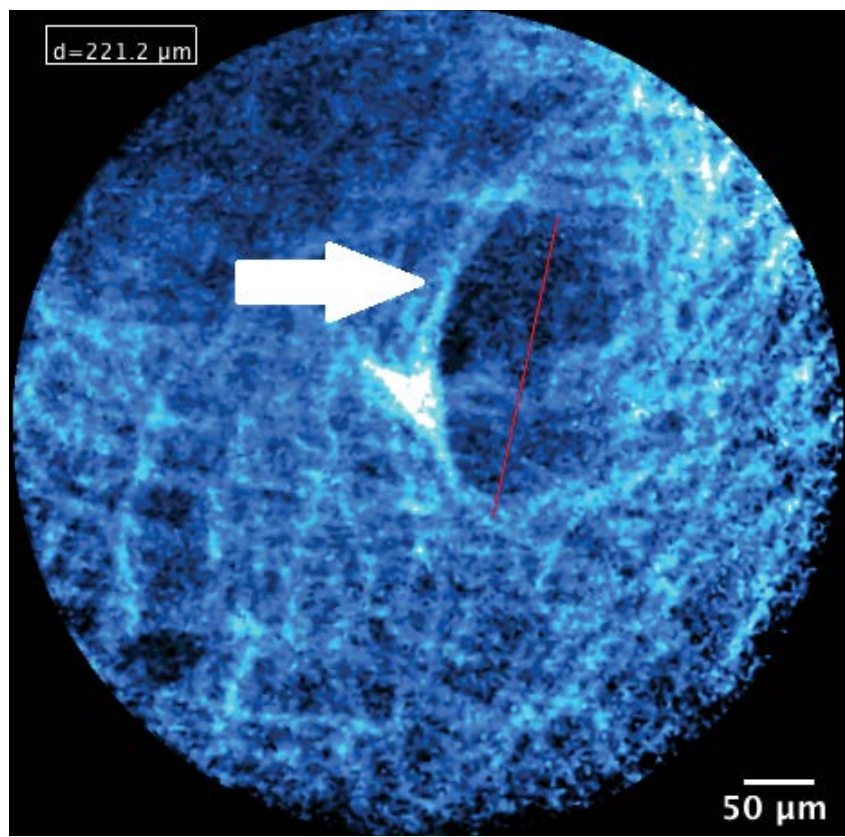

Figure 7: Bronchial gland opening (white arrow) with a network of tightly compacted, crossing fibers.

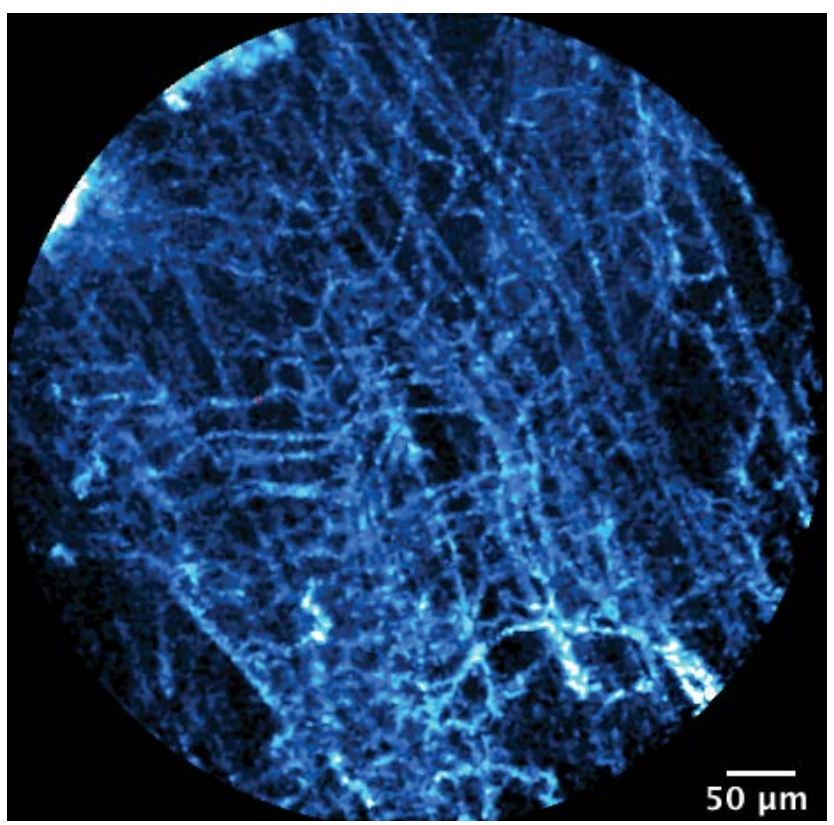

Figure 8: Bronchiole with an interlacing lattice of $2 \mu \mathrm{m}$ to $5 \mu \mathrm{m}$ fibers.

Topical acriflavine $(10 \mathrm{~mL}$ of a $0.05 \%$ solution) is an alternative contrast agent. It can be administered via the working channel of the bronchoscope 2 minutes prior to pCLE [20]. These 2 minutes allow even distribution and cellular uptake such that the nuclei of epithelial cells are seen. The confocal images that were captured can be classified into three different categories: normal tissue, inflammation/regeneration and neoplasia [20]. Normal airway mucosa has homogeneous tissue architecture with bright nuclei and overlapping of cells. In contrast, neoplastic lesions have heterogeneous architecture with areas of poor acriflavine uptake. There is no overlapping and the cell distribution is chaotic. Furthermore, the nuclei are of different sizes with variable

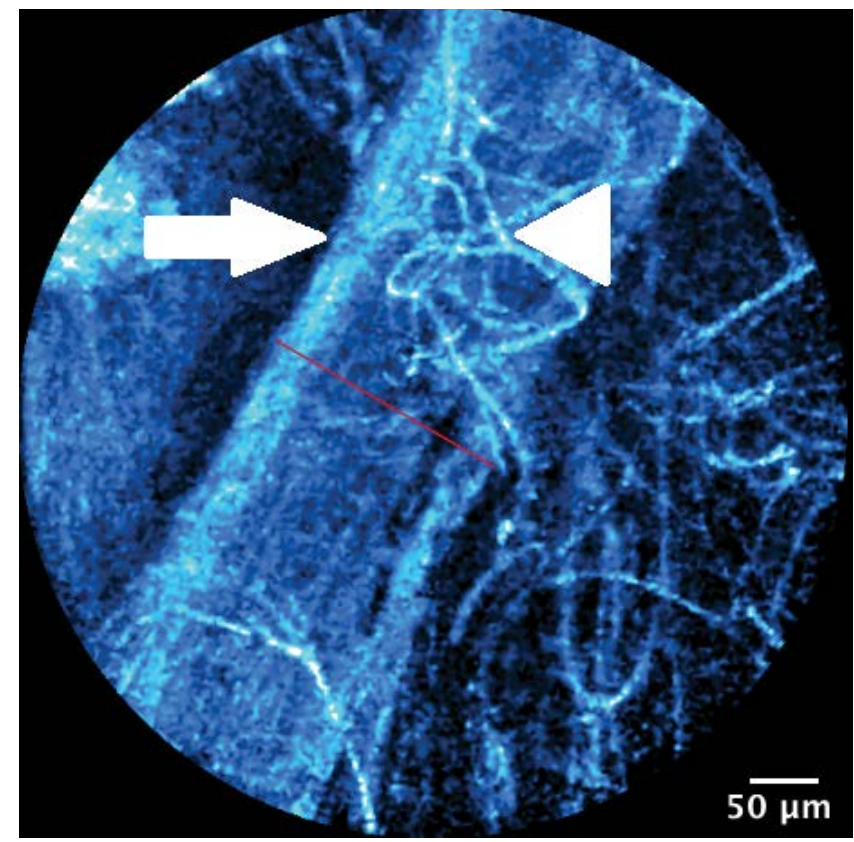

Figure 9: Micro vessel (white arrow) in the distal airspaces. Broken fibers (white arrow head) secondary to miniprobe trauma are also seen.

distances between them. Inflammatory lesions/regenerative tissues tend to have expanded cytoplasm with heterogeneous architecture and no overlapping cells. In airway lesions, neoplastic changes can be predicted using these criteria with a sensitivity of $96 \%$ and specificity of $87 \%$ [20]. However, caution is advised because animal studies have suggested that acriflavine is carcinogenic [21]. Methylene blue is another topical contrast agent and is able to highlight both the nucleus and cytoplasm. It has a better safety profile, but requires illumination under a different light wavelength $(660 \mathrm{~nm})$ [21,22].

Any attempt to evaluate peripheral lung lesions, especially early stage neoplasm necessitates the use of navigational bronchoscopic technology such as radial endobronchial ultrasound to ensure that imaging and biopsy are obtained from the same location within the thorax [23]. Preliminary data shows that malignant lesions tend to have alteration in alveolar structure; compact and thickened alveoli $[24,25]$; disruption of elastin around the alveolar walls; disorganized clumping of tissue; and sometimes, a 'Swiss cheese' appearance of nests of tumor [26]. There may also be stippling along the edge of clumped tissue suggesting a lepidic growth pattern [26]; mottled or 'black hole' appearance of the microvessels caused by shadow effect of tumor cells [27]; and thickened, irregular fibers in the interstitium [28]. In contrast, infected areas have preserved alveolar architecture, normal alveolar wall thickness and alveolar spaces filled with inflammatory cells [29]. These patterns are currently being tested to evaluate the sensitivity in diagnosing malignancy using pCLE [23]. Computer-aided diagnosis is also being developed to perform the quantitative analysis using alveolar diameter, vessel size and optical density to differentiate malignant from benign lesions [30]. With administration of intravenous fluorescein sodium, dark neoplastic and inflammatory cells are visible in the distal airspaces in peripheral lung regions $[18,19]$. However, due to the local detergent effect, foam-like structures are quickly formed and block the view of alveolar structures [18].

\section{Airway diseases}

Three patterns of airway wall elastin fibres have been identified: 
lamellar, loose and mixed [13]. The lamellar pattern is characterised by a linear and parallel bronchial thick fibre orientation. The loose pattern is described as wispy i.e. no specific pattern with some occasional thin lines that lack a specific orientation [31]. There is good agreement between elastin fibre patterns on histology and pCLE images with a linearly weighted kappa 0.744 [31]. However, no specific elastin fibre pattern was detected in patients with asthma. Subjects with a laminar pattern tend to have lower post-bronchodilator forced expiratory volume in one second percentage predicted. Further studies with more severe asthma may show the fragmentation of fibres observed in histological study [32]. The loose pattern is 3 times more prevalent in patients with chronic obstructive pulmonary disease (17\%) than in controls (5\%) [13].

Besides elastin fibre pattern, other structural changes corresponding to lung function abnormalities in chronic obstructive pulmonary disease have been found [13]. There appears to be a correlation between the small vessel diameter and the carbon monoxide transfer coefficient $(\mathrm{r}=0.388, \mathrm{p}=0.044)$. The alveolar diameter has been visualized to be significantly larger in emphysema $(322.4 \mu \mathrm{m} \pm 45.8 \mu \mathrm{m})$ compared to both smokers $(282.2 \mu \mathrm{m} \pm 42.2 \mu \mathrm{m})$ and non-smokers $(267.8 \mu \mathrm{m}$ $\pm 54 \mu \mathrm{m})$. This increase in alveolar size has been corroborated by other studies [11,33]. The alveolar diameter was inversely related to measures of airflow limitation such as the forced expiratory volume in 1 second $(r=-0.338, p=0.033)$ [13]. This opens new possibilities for in vivo assessment of therapeutic interventions in COPD, such as bronchoscopic lung volume reduction.

In patients post lung transplantation, the combination of the autofluorescent cell count, the intensity of cellular fluorescence and vascular index (ratio between the thickness of the autofluorescent lining of a vessel and its diameter) has a sensitivity of $93 \%$ and a specificity of $83 \%$ of diagnosing acute rejection [12]. To avoid confounding this finding with smoking status, the data excluded current smokers and those who had ever smoked for the preceding 6 months. The autofluorescent cell count ( $>19$ cells per microscopic field) alone had both a sensitivity and specificity of 79\% [12]. Another study shows that perivascular cellular infiltration in patients with clinical signs of acute rejection has a sensitivity of $83 \%$ and specificity of $94 \%$ for diagnosing acute rejection [34]. By comparison, conventional transbronchial lung biopsy has a sensitivity ranging from $72 \%$ to $84 \%[35,36]$. The histopathological basis for these findings in acute rejection has not yet been explained especially since pCLE cannot visualise lymphocytic airway infiltration in other studies.

\section{Diffuse parenchymal lung disease}

In diffuse parenchymal lung disease, elastin autofluorescence is reduced and the images can become indistinct [11]. The worst affected areas on radiology have the most disruption of normal architecture and loss of autofluorescence. Single lung transplant recipients have facilitated comparison between the transplanted lung and the remaining diseased lung [10]. In idiopathic pulmonary fibrosis $(n=28)$, the alveolar diameter is smaller $(228 \mu \mathrm{m} \pm 68 \mu \mathrm{m})$ compared to the transplanted lung $(258 \mu \mathrm{m} \pm 42 \mu \mathrm{m})$ [10]. The age groups of the donors and recipients were well matched in this study. Elastin fiber thickness is greater $(15.9 \mu \mathrm{m} \pm 5 \mu \mathrm{m})$ in idiopathic pulmonary fibrosis compared to $10.2 \mu \mathrm{m} \pm 3 \mu \mathrm{m}$ in the transplanted lung [10]. In other case reports of idiopathic pulmonary fibrosis, the elastin fibers were also found to be thickened and rigid with higher concentration. In addition, the alveolar architecture was indistinct (Figure 10) [37-39]. It remains unclear as to how various radiological patterns such as ground glass opacification, reticulation and honey comb appearance affect pCLE imaging.
Amiodarone pneumonitis and pulmonary alveolar proteinosis are two diffuse parenchymal lung diseases where pCLE can produce distinct images. The presence of at least one alveolar area with large $(>20 \mu \mathrm{m})$ and strongly fluorescent cells has a sensitivity of $100 \%$ and a specificity of $88 \%$ in the diagnosis of amiodarone induced pneumonitis [40]. These cells appear to correspond to foamy macrophages. In patients with pulmonary alveolar proteinosis, fluorescent floating complexes ( $100 \mu \mathrm{m}$ to $450 \mu \mathrm{m}$ ) were found in $74.4 \%$ (90 out of 121 ) of alveolar areas explored [41]. Cytologic examination of bronchoalveolar lavage fluid showed clustered alveolar macrophages and characteristic globular lipoproteinaceous material that corresponded in size and morphology to the pCLE images [42]. Alveolar acinar structures were preserved and the fluorescent complexes appeared to be floating in a slightly fluorescent alveolar fluid. Even in the segments which had no high resolution computed tomography features of alveolar proteinosis, fluorescent complexes could still be found suggesting superior sensitivity to radiology. In addition, after whole lung lavage, these complexes were clearly reduced based on a semi-quantitative estimation [41]. However, the majority of cases of pulmonary alveolar proteinosis were also smokers with clearly visible alveolar macrophages $[41,42]$. The reproducibility of these distinct features in non-smokers has yet to be shown.

\section{Future Direction}

Bronchoscopic lung biopsy can be complicated by pneumothorax (1\% to 6\%) and bleeding (1.9\%) [43]. Specimens obtained by forceps are subjected to crush artifact and biopsied areas often undergo scarring thus altering the natural history of disease. Processing of tissues involves dehydration and chemical fixation that result in specimens not being analyzed in their natural state. The risks of bronchoscopic biopsy and the limitations of specimen processing have fueled the development of in vivo optical biopsies. This will enable non-invasive diagnosis and monitoring of both treatment response and disease progression.

The pCLE shows potential to deliver optical biopsies, but there are limitations that should be addressed. Diagnostic ability is greatly

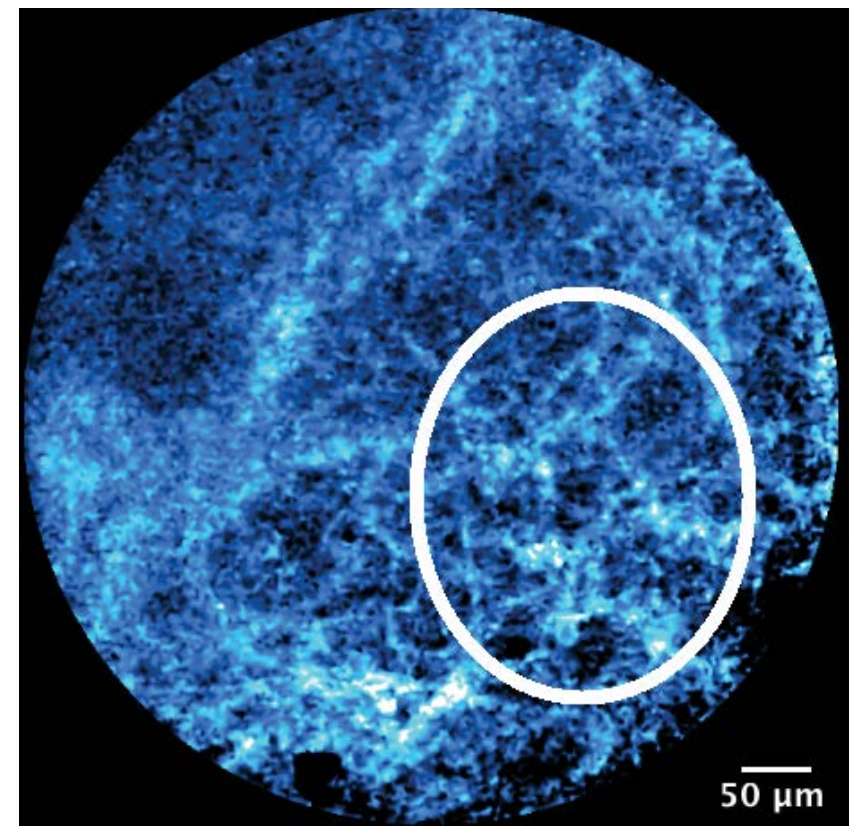

Figure 10: Idiopathic pulmonary fibrosis with thickened, rigid and highly concentrated fibers (white circle). The alveolar architecture is indistinct. 
limited by only being able to visualize elastin. Use of contrast agents or activation light of alternative wavelengths need to be developed. Recent animal studies using fluorescein isothiocyanate labelled erythrocytes can overcome the foaming problems in fluorescein aided pCLE and clearly visualize capillary networks with alveolar structures $[18,44]$. A ventilating ex vivo asinine lung model with optical lysyl oxidase probe has shown real-time lysyl oxidase activity which is an indicator of lung fibrosis [45]. Additionally the field of view is extremely small and better targeting with other bronchoscopic technology such as navigation bronchoscopy, narrow-band imaging or autofluorescence bronchoscopy should be considered. Miniprobes with less stiffness will enable consistent visualization of all bronchopulmonary segments. Endoscopists will also need to reach a consensus on standardization of pCLE procedure, image acquisition and analysis to facilitate further comparison between patients. These developments can then unlock the true potential of pCLE.

\section{References}

1. Minsky M (1957) Microscopy apparatus. US Patent US3013467

2. Jabbour JM, Saldua MA, Bixler JN, Maitland KC (2012) Confocal endomicroscopy: instrumentation and medical applications. Ann Biomed Eng 40: $378-397$.

3. Wang KK, Carr-Locke DL, Singh SK, Neumann H, Bertani H, et al. (2015) Use of probe-based confocal laser endomicroscopy ( $p C L E)$ in gastrointestinal applications. A consensus report based on clinical evidence. United Eur Gastroenterol J 3: 230-254.

4. ASGE Technology Committee, Komanduri S, Abu Dayyeh BK, Bhat YM, Chauhan SS, et al. (2014) Technologies for monitoring the quality of endoscope reprocessing. Gastrointest Endosc 80: 369-373.

5. Musani Al, Sims M, Sareli C, Russell W, McLaren WJ, et al. (2010) A pilot study of the feasibility of confocal endomicroscopy for examination of the human airway. J Bronchology Interv Pulmonol 17: 126-130.

6. Thiberville L, Salaün M, Lachkar S, Dominique S, Moreno-Swirc S, et al. (2009) Human in vivo fluorescence microimaging of the alveolar ducts and sacs during bronchoscopy. Eur Respir J 33: 974-985.

7. Filner JJ, Bonura EJ, Lau ST, Abounasr KK, Ost D, et al. (2011) Bronchoscopic fibered confocal fluorescence microscopy image characteristics and pathologic correlations. J Bronchol Intervent Pulmonol 18: 23-30.

8. Rafeq S, Ernst A, Majid A, Michaud G, Reddy C, et al. (2009) Bronchoscopic imaging using fibered confocal fluorescence microscopy. Am Thoracic Soc Int Conf Abs 2009: A5772.

9. Bhatt NA, Parrish SC, Malafronte P, Browning RF (2015) Characterization of pleura by probe-based confocal laser endomicroscopy. Am Thoracic Soc Int Conf Abs 2015: A4971.

10. Keller C, Alvarez F, Erasmus D, Khoor A, Shahid M, et al. (2011) Probe-based confocal laser endomicroscopy ( $\mathrm{pCLE}$ ) in lung transplantation. Proc Int Conf Cellvizio ${ }^{\circ}$ Users.

11. Newton RC, Kemp SV, Yang GZ, Elson DS, Darzi A, et al. (2012) Imaging parenchymal lung diseases with confocal endomicroscopy. Respir Med 106: 127-137.

12. Yserbyt J, Dooms C, Decramer M, Verleden GM (2014) Acute lung allograft rejection: diagnostic role of probe-based confocal laser endomicroscopy of the respiratory tract. J Heart Lung Transplant 33: 492-498.

13. Cosío BG, Shafiek H, Fiorentino F, Gómez C, López M, et al. (2014) Structurefunction relationship in COPD revisited: an in vivo microscopy view. Thorax 69: 724-730.

14. Thiberville L, Moreno-Swirc S, Vercauteren T, Peltier E, Cavé C, et al. (2007) In vivo imaging of the bronchial wall microstructure using fibered confocal fluorescence microscopy. Am J Respir Crit Care Med 175: 22-31.

15. Weibel ER, Sapoval B, Filoche M (2005) Design of peripheral airways for efficient gas exchange. Respir Physiol Neurobiol 148: 3-21.

16. Thiberville L, Salaün M, Bourg-Heckly G (2010) In vivo confocal microendoscopy: from the proximal bronchus down to the pulmonary acinus. Russian J Transplantol Artif Organs 15: 81-108.
17. Pauly JL, Allison EM, Hurley EL, Nwogu CE, Wallace PK, et al. (2005) Fluorescent human lung macrophages analyzed by spectral confocal laser scanning microscopy and multispectral cytometry. Microsc Res Tech 67: 79-89.

18. Fuchs FS, Zirlik S, Hildner K, Frieser M, Ganslmayer M, et al. (2011) Fluorescein-aided confocal laser endomicroscopy of the lung. Respiration 81 : 32-38.

19. Shibuya K, Okada N, Horio J, Funabashi H, Iwai N, et al. (2015) Fluoresceinaided probe-based confocal laser endomicroscopy (PCLE) for detection of peripheral lung and central airways carcinoma. Proc Int Conf Cellvizioß Users.

20. Fuchs FS, Zirlik S, Hildner K, Schubert J, Vieth M, et al. (2013) Confocal lase endomicroscopy for diagnosing lung cancer in vivo. Eur Respir J 41: 14011408.

21. Obstoy B, Salaun M, Veresezan L, Sesboüé R, Thiberville L, et al. (2015) Safety and performance analysis of acriflavine and methylene blue for in vivo imaging of precancerous lesions using fibered confocal fluorescence microscopy (FCFM): an experimental study. BMC Pulm Med 15: 30.

22. Thiberville L, Salaün M, Lachkar S, Dominique S, Moreno-Swirc S, et al. (2009) In vivo confocal fluorescence endomicroscopy of lung cancer. Proc $13^{\text {th }}$ World Conf Lung Cancer.

23. Wellikoff A, Islam S, Browning R, Khurana S, Wilson D, et al. (2014) Probebased confocal laser endomicroscopy in the diagnosis of discrete pulmonary lesions: results of a phase 1 multi-center prospective study. Proc Am Thoracic Soc Int Conf Abs 2014: A2510.

24. Arenberg DA, Gildea T, Wilson D (2011) Proposed classification of probe-based confocal laser endomicroscopy (PCLE) findings for evaluation of indeterminate peripheral lung nodules. Proc Am Thoracic Soc Int Conf Abs 2011: A6097.

25. Li S, Luo W, Zhong G, Chen X, Chen Y, et al. (2015) Human in vivo imaging of lung cancer using probe-based confocal laser endomicroscopy. Proc Int Conf Cellvizio® Users.

26. Holladay RC, Turbat-Herrera EA, (2015) Comparison of pCLE images with histopathology in the lung-Focus on adenocarcinoma. Proc Int Conf Cellvizio® Users.

27. Wellikoff AS, Holladay RC, Downie GH, Chaudoir CS, Brandi L, et al. (2015) Comparison of in vivo probe-based confocal laser endomicroscopy with histopathology in lung cancer: A move toward optical biopsy. Respirology 20: 967-974.

28. Kurimoto N, Fujiwara M, Miyazawa T (2015) Usefulness of characteristics of fibers of elastin network of lung tissue visualized by probe-based confocal laser endomicroscopy. Proc Int Conf Cellvizioß Users.

29. Lee P, Seet JE (2013) Confocal laser endomicroscopy (pCLE) for evaluation of pulmonary nodule and mass. Proc Int Conf Cellvizio® Users.

30. Yang D, Ye M, Yin X, Bai C, Hu J (2015) Clinical application of probe-based confocal laser endoscopy (pCLE) in diagnosis of pulmonary diseases. Proc Int Conf Cellvizioß Users.

31. Yick CY, von der Thüsen JH, Bel EH, Sterk PJ, Kunst PW (2011) In vivo imaging of the airway wall in asthma: fibered confocal fluorescence microscopy in relation to histology and lung function. Respir Res 12: 85.

32. Bousquet J, Lacoste, JY, Chanez P, Vic P, Godard P, et al. (1996) Bronchia elastic fibers in normal subjects and asthmatic patients. Am J Respir Crit Care Med 153: 1648-1654.

33. Salaün M, Modzelewski R, Marie JP, Moreno-Swirc S, Thiberville L, et al. (2012) In vivo assessment of the pulmonary microcirculation in elastaseinduced emphysema using probe-based confocal fluorescence microscopy. Intravital 1: 122-131.

34. Keller C, Hurst K, David-Robinson H, Alvarez F, Erasmus D, et al. (2015) Probebased confocal laser endomicroscopy (PCLE) in lung transplant recipients with symptoms consistent with acute rejection. Proc Int Conf Cellvizio ${ }^{\circledR}$ Users.

35. Trulock EP, Ettinger NA, Brunt EM, Pasque MK, Kaiser LR, et al. (1992) The role of transbronchial lung biopsy in the treatment of lung transplant recipients. An analysis of 200 consecutive procedures. Chest 102: 1049-1054.

36. Higenbottam T, Stewart S, Penketh A Wallwork J (1988) Transbronchia lung biopsy for the diagnosis of rejection in heart-lung transplant patients. Transplantation 46: 532-539.

37. Wellikoff A, Brandi L, Small C, Pea S, Holladay R (2013) Probe-based confocal laser endomicroscopy imaging of interstitial lung disease. Proc Am Thoracic Soc Int Conf Abs 2013: A5796. 
Citation: Peng M, Liang TG, Anantham D (2016) Probe-Based Confocal Laser Endomicroscopy of the Lungs. J Pulm Respir Med 6: 373. doi: 10.4172/2161-105X.1000373

38. Dalar L, Özdemir C, Sökücü SN, Karasulu AL, Altin S, et al. (2014) Probe-based confocal Laser endomicroscopy (pCLE) in the diagnosis of diffuse parenchymal lung diseases: two cases. Austin J Pulm Respir Med 1: 1018.

39. Reichenberger F, Silbernagel E, Gesierich W, Lindner M, Morresi-Hauff A, et al. (2015) Imaging pattern in interstitial lung diseases using probe based confocal laser endomicroscopy (PCLE). Proc Int Conf Cellvizio® Users.

40. Salaün M, Roussel F, Bourg-Heckly G, Vever-Bizet C, Thiberville L, et al. (2013) In vivo probe-based confocal laser endomicroscopy in amiodarone-related pneumonia. Eur Respir J 42: 1646-1658.

41. Danilevskaya O, Averyanov A, Lesnyak V, Chernyaev A, Sorokina A (2015) Confocal laser endomicroscopy for diagnosis and monitoring of pulmonary alveolar proteinosis. J Bronchology Interv Pulmonol 22: 33.
42. Salaün M, Roussel $F$, Hauss $P A$, Lachkar $S$, Thiberville $L$ (2010) In vivo imaging of pulmonary alveolar proteinosis using confocal endomicroscopy. Eur Respir J 36: 451-453.

43. du Rand IA, Blaikley J, Booton R, Chaudhuri N, Gupta V, et al. (2013) British Thoracic Society guideline for diagnostic flexible bronchoscopy in adults. Thorax 68: i1-i44.

44. Gruber S, Spielauer I, Böhme S, Baron D, Klein KU, et al. (2015) Real-time in-vivo imaging of pulmonary capillary perfusion using probe-based confocal laser scanning endomicroscopy in pigs: An interventional laboratory study. Eur J Anaesthesiol 32: 392-399.

45. Aslam T, Miele A, Chankeshwara SV, Bradley M, Dhaliwal K, et al. (2015) Optical molecular imaging of lysyl oxidase activity-detection of active fibrogenesis in human lung tissue. Chem Sci 6: 4946-4953. 